Colette FEUILLARD

Université René Descartes-Paris 5

\title{
LA DYNAMIQUE SYNCHRONIQUE DANS LES LANGUES
}

Les linguistes depuis fort longtemps ont mentionné le caractère mouvant des langues, et Saussure lui-même dans ses Ecrits de linguistique générale, plus précisément lors de sa deuxième conférence à l'université de Genève en novembre 1891, note qu' « il n'y a jamais ... un équilibre, un point permanent, stable dans aucun langage », ce qui l'a amené à poser le principe de «la transformation incessante des langues comme absolu ». Il précise encore que « le cas d'un idiome qui se trouverait «en état d'immobilité et de repos ne se présente pas» (SAUSSURE, $2002: 158$ ).

Mathesius dans son article intitulé «On the Potentiality of the Phenomena of Language » de 1911 (VACHEK, 1964 : 1), insiste également sur la mouvance des faits linguistiques. Il établit une distinction essentielle entre la potentialité des phénomènes du langage qui se manifeste par des oscillations statiques et la changeabilité dynamique qui se traduit par des altérations d'une langue donnée dans le temps.

Bally dans Le langage et la vie de 1913 insiste sur le fait que "personne ne doute plus que les langues changent d'une façon continue » et que « cette certitude est une grande conquête de la linguistique » (BALLY, 1952:34).

Frei dans La grammaire des fautes, dont la première édition date de 1929, montre que les erreurs commises par les locuteurs obéissent aux principes de fonctionnement des langues (analogie, besoin de brièveté, etc.). En effet, « un fait de langage a beau être correct, il peut ne pas être adapté à sa fonction » et « un très grand nombre d'incorrections, peut-être la majorité, servent ... à prévenir ou à réparer de tels déficits » (FREI, $1971: 18-19)$.

Enfin, plus près de nous, Martinet dans Économie des changements phonétiques, ouvrage publié la première fois en 1955, insiste sur la dynamique linguistique et refuse une coupure radicale entre diachronie (ou étude de l'évolution d'une langue dans le temps) et synchronie (étude d'un état de langue à un moment donné de son évolution). Il préfère opposer à l'étude diachronique, qui « vise délibérément à comparer différents états successifs du même objet d'étude, une synchronie dynamique, où l'attention se concentre, certes, sur un même état de langue, mais sans qu'on renonce jamais à y relever des variations et à y évaluer le caractère progressif ou récessif de chaque trait» (MARTINET, 1975: 9); il considère qu' « une langue change à tous les instants parce qu'elle fonctionne » (MARTINET, 1990 : 13).

Le rappel de ces différents points de vue montre à quel point la mouvance des langues est prégnante chez les linguistes de la fin du $\mathrm{XIX}^{\mathrm{e}}$ siècle et du XX $\mathrm{X}^{\mathrm{e}}$ jusqu'à nos jours. Il ne sera pas question, ici, de comparer ces diverses prises de position afin de déterminer si la variabilité des faits linguistiques est envisagée de manière analogue et se situe aux mêmes niveaux d'analyse chez tous les auteurs. L'étude proposée se centrera principalement sur l'analyse du concept et des phénomènes d'oscillation et de potentialité chez Mathesius, sur la notion et les faits de dynamique synchronique dans l'optique fonctionnelle, tels que je les conçois, à 
partir d'exemples empruntés au français. Puis, dans un dernier temps seront examinés les rapports susceptibles d'être établis entre les oscillations et les variations $^{1}$ relevant de la dynamique synchronique. Si cette expression de dynamique synchronique est utilisée de préférence à celle de synchronie dynamique, c'est parce qu'elle me semble beaucoup mieux mettre en lumière la variation et le processus qui la sous-tend, le concept de synchronie dynamique insistant davantage sur la perspective d'analyse.

\section{DÉFINITION DES CONCEPTS D'OSCILLATION ET DE DYNAMIQUE}

\section{1. Caractérisation générale}

L'oscillation correspond à un «mouvement d'un corps qui se déplace alternativement et plus ou moins régulièrement de part et d'autre d'une position d'équilibre » (TLF).

La dynamique concerne le «mouvement produit par des forces». Ce mouvement implique "des modifications incessantes selon une finalité ». En sociologie, il s'agit de "l'étude des forces créatrices auxquelles on attribue une valeur causale dans l'évolution et le progrès des sociétés » (TLF).

\section{2. Confrontation de ces définitions et implications en linguistique}

Alors que l'oscillation, telle qu'elle est caractérisée sur le plan général, suppose une variation autour d'un point d'équilibre, c'est-à-dire d'un axe fixe, qui pourrait être, en linguistique, les unités de la langue en tant qu'entités du système, la dynamique implique un mouvement entraîné par des forces, visant dans certains cas à une finalité, qui, notamment en sciences sociales, peut être envisagée en termes d'évolution et de progrès.

Cette dynamique peut donc se réaliser sur l'axe de la successivité, lorsqu'il y a évolution du système. On est alors en diachronie, puisqu'il y a passage d'un état de langue à un autre. Elle peut encore se manifester sur l'axe des simultanéités. Il s'agit, dans ce cas, de la dynamique synchronique. Afin de conférer à l'analyse une cohérence interne, c'est cette dernière perspective qui sera retenue, dans la mesure où les oscillations se manifestent en synchronie.

\section{OSCILLATION ET POTENTIALITE CHEZ MATHESIUS (1911)}

\section{1. Les domaines d'oscillation}

Les oscillations peuvent affecter, en particulier,

$>$ le domaine phonétique :

\footnotetext{
${ }^{1}$ Le terme de variation a une valeur très générale et peut désigner, dans l'absolu, aussi bien des oscillations que des faits de dynamique synchronique. Néanmoins, pour différencier ces deux types de phénomènes, il ne référera, dans le cadre de cet exposé, qu'aux faits de dynamique synchronique.
} 
Ainsi, la réalisation d'un son dans des mots prononcés isolément est susceptible de varier non seulement chez un même locuteur, mais aussi en fonction des unités concernées, et de leur environnement phonique. Il en va de même dans la phrase. Néanmoins, le domaine de réalisation de ces oscillations est limité et variable selon les sons (MATHESIUS, $1911: 3-9$ ) ;

$>$ le domaine morphologique :

Ces oscillations, à l'oral, peuvent toucher la forme des mots, plus précisément leur indépendance formelle au sein de la phrase, tout comme la forme des phrases elles-mêmes, notamment la place des enclitiques (MATHESIUS, 1911 : 9-16). Elles concernent encore l'intensité de l'accentuation de certaines catégories grammaticales, ainsi que l'ordre des mots dans la phrase (MATHESIUS, 1911 : 1621);

$>$ le domaine sémantique :

Elles peuvent porter soit sur le sens d'une unité donnée, soit sur la possibilité d'exprimer une même idée sous des formes différentes (MATHESIUS, 1911 : 2122) : cf. gifler et donner une gifle.

\section{2. La diversité des plans d'oscillation}

$>$ Certaines oscillations portent sur les unités du système, en tant qu'entités :

Il s'agit des sons et du sens des unités prises individuellement. La variation d'intensité de l'accent des catégories grammaticales évoquée par Mathesius ne concerne pas le système lui-même, puisque seule la position de l'accent est susceptible d'être pertinente en linguistique et non l'accent en tant que tel.

$>$ D'autres concernent la structure du système :

C'est le cas notamment de la coexistence dans une langue donnée d'expressions susceptibles de renvoyer à une même idée, pour reprendre les termes de Mathesius.

$>$ D'autres encore sont déterminées par le mode de réalisation du discours :

En effet, le problème de l'indépendance formelle des mots tel qu'il est présenté par Mathesius ne se pose qu'à l'oral, l'écrit n'étant pas évoqué.

$>$ L'ordre des éléments dans la phrase n'a pas un statut fixe :

Il ne s'agit pas d'une unité du système en soi. L'ordre résulte de la linéarité du discours. Néanmoins, il peut être utilisé à des fins linguistiques, c'est-à-dire de communication, quand il est pertinent, Pierre admire Paul, Paul admire Pierre. 
Il arrive également qu'il soit imposé sans que cela ait une incidence quelconque sur le sens. C'est le cas des déterminants temporels de forme simple qui sont joints au verbe en français, comme le futur, il viendra.

Que la position d'une unité soit pertinente ou qu'elle soit imposée, elle est nécessairement fixe et ne peut donc subir d'oscillations. En revanche, celles-ci deviennent éventuellement possibles quand elles n'ont aucune conséquence sur la communication, aucun rôle linguistique ${ }^{2}$ n'étant alors attribué à l'ordre. On pourrait tout au plus leur conférer une valeur stylistique.

\section{3. Caractéristiques des oscillations}

Elles peuvent être individuelles ou générales. Leur domaine de réalisation est circonscrit et varie d'une langue à l'autre.

Par ailleurs, au terme de son étude, Mathesius pose explicitement le problème de savoir à quel moment les oscillations renvoient à un seul et même phénomène ou au contraire à un phénomène nouveau (MATHESIUS, $1911: 31$ ).

\section{4. Causes des oscillations}

D'après Mathesius, elles sont liées à la potentialité des phénomènes linguistiques. Cette potentialité pourrait être expliquée, me semble-t-il, par les dichotomies inspirées de Saussure, puis reprises et développées par Hjelmslev dans Prolégomènes à une théorie du langage (1971 : 71-85) entre expression et contenu d'une part, forme et substance d'autre part, ou plus précisément entre forme de l'expression et du contenu, " fonctifs qui contractent la fonction sémiotique » (HJEMSLEV, 1971: 72), et substance de l'expression et du contenu, même si ces distinctions ont été proposées postérieurement à la notion d'oscillation. En effet, toute langue opère un découpage du continuum phonique (substance de l'expression) et de l'expérience (substance du contenu) en unités abstraites (forme de l'expression, phonèmes et signifiants des monèmes ${ }^{3}$ et forme du contenu, signifié des monèmes), dont certaines sont discrètes, à savoir les phonèmes et les signifiants. Étant abstraites et du domaine de la forme, ces unités linguistiques sont potentielles et identifiables, donc stables à un moment donné, sinon, il n'y aurait pas de système, mais elles doivent, pour se réaliser, recourir au matériau phonique et référer à l'expérience à communiquer. Leur réalisation implique donc le recours à la substance de la forme et du contenu, qui, elle, est continue, ce qui explique que l'oscillation puisse être aussi bien individuelle que générale. En outre, leur concrétisation dans le discours est nécessairement influencée par le contexte dans lequel les unités sont insérées ; étant imposé alors par des facteurs linguistiques, le phénomène d'oscillation prend nécessairement, ici, une valeur générale. Ainsi, le phonème /i/ placé entre deux occlusives bilabiales /p/, comme dans /pip/ «pipe », se réalise-t-il plus en avant que lorsqu'il est entouré d'une vélaire $/ \mathrm{k} /$ et d'une uvulaire $/ \mathrm{R} /, / \mathrm{kiR} /$ «kir », compte tenu du lieu de réalisation de chacune de ces consonnes.

\footnotetext{
${ }^{2}$ Une unité est considérée comme linguistique quand elle répond à la fonction de communication.

${ }^{3}$ Il s'agit de l'unité significative minimum, qui, dans d'autres courants théoriques est appelée morphème.
} 
Les unités du système linguistique, par nature potentielles puisqu'elles sont abstraites, constitueraient les axes à partir desquels s'effectueraient les oscillations lors de leur concrétisation dans le discours ${ }^{4}$, les oscillations correspondant aux latitudes de réalisation des unités concernées. Toutefois, comme cela a été mentionné précédemment, Mathesius ne se limite pas à l'examen des seules unités du système. Il semble élargir cette notion de potentialité, entre autres, au choix du locuteur, en particulier lorsqu'il mentionne la possibilité d'exprimer une même idée de plusieurs manières ou lorsqu'il évoque l'ordre. Le concept de potentialité recouvre alors des valeurs différentes; dans le cas des unités, cf. /i/, la potentialité leur est inhérente, mais quand il réfère au choix du locuteur, cette potentialité résulte tantôt de la structure du système, plus précisément de la coexistence dans la langue de formes différentes pour l'expression d'une même donnée de l'expérience, cf. gifler, donner une gifle, tantôt de la liberté positionnelle de certains éléments, laquelle est le plus souvent liée au rôle syntaxique qui leur est assigné dans la phrase : si l'on peut estimer que la position de hier dans hier, il est allé au cinéma, il est allé au cinéma, hier est libre, bien que l'on puisse se demander si la visée communicative est la même dans les deux cas, elle est absolument fixe dans il nous a raconté sa journée d'hier en raison de la fonction exercée par hier à l'égard de journée.

\section{5. Conséquences des oscillations}

Certaines peuvent être source de difficultés de délimitation et d'identification et par suite de compréhension pour l'allocutaire, puis rétroactivement d'analyse pour le descripteur.

C'est le cas, en particulier, de l'oscillation des sons, du sens des unités, et de la forme des mots à l'oral.

D'autres, en revanche, n'ont aucune incidence, ni sur le plan de la compréhension, ni sur celui de l'analyse.

Ce sont celles qui relèvent notamment de l'intensité de l'accent, des possibilités de choix au sein du système pour exprimer une même expérience, ou de l'ordre ${ }^{5}$. Or, ces trois types d'oscillations ne concernent pas les unités ellesmêmes.

En conséquence, et compte tenu des objectifs fixés au départ, l'étude se limitera à une comparaison entre les phénomènes d'oscillation affectant les unités linguistiques et les faits de dynamique synchronique.

\footnotetext{
${ }^{4}$ Le terme discours est pris dans son sens général et renvoie aussi bien à la forme écrite qu'orale de la réalisation effective de la langue dans une situation déterminée.

${ }^{5}$ Cela ne vaut, dans une large mesure, que si la visée communicative n'est pas prise en considération. 


\section{DYNAMIQUE SYNCHRONIQUE DANS LES LANGUES}

\section{1. Définition}

En référence à la perspective fonctionnelle, telle qu'elle a été développée par André Martinet, j'entends par dynamique synchronique les variations, en un temps donné et dans une langue déterminée, des unités linguistiques dans leur identité, c'est-à-dire en tant qu'entités du système, ce qui implique, pour le linguiste, la prise en compte de l'ensemble de leurs propriétés et la référence à une norme dite « standard ${ }^{6}$, transmise par l'enseignement et qui, de ce fait, implique un certain consensus de la part des locuteurs.

\section{2. Éléments concernés}

Ces unités peuvent être des phonèmes, des monèmes ou des fonctions syntaxiques. Ces variations peuvent affecter soit l'unité dans sa totalité, soit son aspect formel, sa valeur significative ou son fonctionnement syntaxique. De plus, elles peuvent interférer, une variation de sens étant susceptible de modifier le comportement fonctionnel d'un élément particulier, et inversement. Il est souvent délicat et parfois impossible de savoir sur quel aspect précis de l'unité a d'abord porté la variation et quelle en est la cause : l'emploi de chez dans le contexte suivant, elle est allée chez Monoprix, de plus en plus fréquent, résulte-t-il de l'extension du sens de la préposition chez, d'une nouvelle valeur attribuée implicitement à ce type de commerce, instaurant un lien de familiarité, voire d'intimité entre le lieu d'achat et le client, ou encore du fait que l'enseigne rappelle un nom propre ? Au contraire, est-ce la combinaison de chez et de Monoprix, deux éléments qui, dans l'usage standard, sont, en principe, incompatibles, chez réclamant un complément pourvu du sème [+ animé] que ne possède pas le terme Monoprix, qui serait à l'origine des variations de sens ou des effets de forme supposés? Il paraît difficile de trancher.

\section{3. Caractéristiques de ces variations}

Elles ne sont

$>$ ni exclusivement individuelles, sinon il s'agirait d'hapax ;

$>$ ni totalement généralisées,

on aurait alors affaire à un nouvel état de langue, qui relèverait de la dynamique diachronique.

Mais elles sont

partiellement collectives.

En effet, elles se répartissent, au sein d'une communauté linguistique donnée, dans des sous-groupes, homogènes ou hétérogènes selon que leurs membres

\footnotetext{
${ }^{6}$ Ce concept ne sera pas développé ici.
} 
présentent ou non des caractéristiques communes, âge, sexe, milieu socio-culturel, etc.

\section{4. Types de variations}

\section{4. 1. Création d'unités linguistiques nouvelles en cours d'intégration}

Les exemples qui vont suivre, machine à laver, lave-vaisselle, lave-linge renvoient à des termes qui sont aujourd'hui parfaitement insérés dans la langue française. Néanmoins, le mot composé lave-linge a été créé après le terme lavevaisselle et par analogie avec ce dernier. En effet, la machine à laver désignait autrefois ce que l'on appelle aujourd'hui le lave-linge. Or, quand le lave-vaisselle est apparu, on a forgé sur la même structure le lave-linge pour spécifier chaque type d'appareils. Lave-linge s'est donc trouvé en concurrence avec machine à laver et l'a progressivement supplanté, sans l'exclure et tout en lui laissant son sens initial. Mais, à terme, on pourrait supposer que machine à laver désignera l'hyperonyme de lave-vaisselle et de lave-linge.

\section{4. 2. Concurrence entre deux ou plusieurs unités et extension d'emploi de l'une au détriment de l'autre}

Ce phénomène peut entraîner ou non la disparition de l'unité concurrencée.

Tendance à la disparition de l'unité concurrencée :

- Phonèmes : /ỗ/, / $/ \tilde{\varepsilon} /$

À l'heure actuelle, le phonème /õ/ qui permet de différencier /bRẽ / «brun »

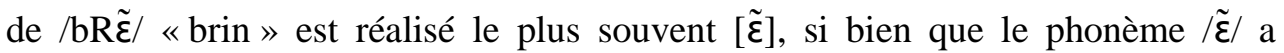
tendance à se substituer à /õe/, les deux unités ne s'opposant l'une à l'autre que par

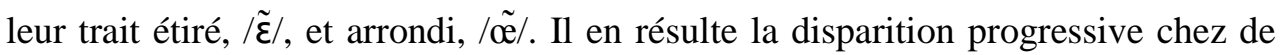
nombreux locuteurs du phonème /ô/ au bénéfice de / $\tilde{\varepsilon} /$.

- Fonctions syntaxiques :

Depuis le $\mathrm{XVIII}^{\mathrm{e}}$ siècle, le verbe se rappeler, qui exige, en principe, la fonction d'objet direct, il se rappelle avec plaisir son séjour aux Antilles, est de plus en plus souvent accompagné de la fonction d'objet indirect, par analogie avec se souvenir dont le sens est très proche, il se rappelle avec plaisir de son séjour aux Antilles. Ce remplacement de la fonction d'objet direct par la fonction d'objet indirect est encore dicté par l'impossibilité d'employer la fonction objet dans certains contextes, * je me te rappelle. On peut donc émettre l'hypothèse qu'un jour la construction indirecte remplacera définitivement la construction directe. Cependant, le poids de la norme institutionnelle est un frein à cette généralisation, et la construction indirecte n'est pas encore totalement intégrée à la langue. 
Maintien, mais restriction d'usage de l'unité concurrencée, la diversité des contextes d'emploi se trouvant réduite :

- Monèmes :

Dans l'exemple mentionné précédemment, elle est allée chez Monoprix, la préposition chez concurrence à, requis par l'usage standard, elle est allée à Monoprix, imposé par l'absence du sème [+ animé] dans le terme Monoprix. Cette utilisation de chez se retrouve avec d'autres unités, elle est allée chez Ikea, puis chez Carrefour.

L'extension de chez au détriment de à et l'élargissement éventuel de sa valeur, ne remet pas en cause pour autant l'existence de la préposition à pas plus qu'elle n'altère son sens, mais elle en limite les contextes d'emploi.

\section{4. 3. Concurrence entre deux ou plusieurs formes d'un même monème et extension d'emploi de l'une d'entre elles au détriment des autres}

Tendance à la disparition de l'une ou de certaines des formes en concurrence :

$\mathrm{Au}$ XVII ${ }^{\mathrm{e}}$ siècle, le verbe «prouver » présentait deux formes concurrentes, prouver et preuver, l'une et l'autre en usage (Dictionnaire LE LITTRÉ, Tome 6 : 563). Cette concurrence a entraîné la disparition de preuver.

Actuellement, la forme du pronom relatif tend à être unifiée, que se substituant fréquemment à dont, c'est une histoire que j'ai entendu parler, et à qui dans certains contextes, la personne qu'est intervenue. Mais les formes standards restent utilisées.

\section{4. 4. Concurrence entre deux ou plusieurs effets de sens d'un même monème}

$>$ Tendance à la disparition ou à l'affaiblissement de la valeur «principale » :

Le terme débile, par exemple, qui désignait au $\mathrm{XIX}^{\mathrm{e}}$ une déficience physique, puis ultérieurement une déficience intellectuelle, est de plus en plus employé dans la langue courante au sens de «stupide», «bête », «idiot». En d'autres termes, il y a eu déplacement des effets de sens au sein du signifié en fonction des situations et des contextes dans lesquels le monème était utilisé.

\section{4. 5. Extension du fonctionnement syntaxique d'un monème}

Dans une phrase telle que question économie, on pourrait mieux faire, question ne fonctionne plus comme un nom, mais comme un thématiseur, susceptible de commuter avec quant à, en ce qui concerne. De plus, il n'est ni expansible, ni déterminable par l'article et le nombre.

Parallèlement à ce comportement syntaxique particulier et relativement nouveau, on peut se demander si question renvoie à un seul et même monème, 
lorsqu'il a le statut de nom et quand il assume le rôle de thématiseur, ou s'il n'y a pas apparition d'une autre entité dont le sens n'a qu'un rapport éloigné avec celui du monème nominal.

\section{5. Procédés mis en jeu par la variation}

Comme le disent Saussure et Bally, il n'y a pas, en langue, de création exnihilo. Aussi la formation de nouvelles unités repose-t-elle le plus souvent sur l'analogie qui «suppose un modèle et son imitation» (FREI, 1971:27), cf. lavelinge et sur le principe d'invariabilité, cf. le pronom relatif.

\section{6. Causes et forces en jeu}

La dynamique synchronique au sein d'une langue n'est pas due au système luimême. Certaines caractéristiques de la structure ou des unités peuvent cependant la favoriser, telles la faible intégration dans le système, comme cela peut être le cas des phonèmes qui n'entrent pas dans une corrélation, le rendement fonctionnel limité d'un élément, cf. /õe/, la multiplicité des formes d'une même unité, qui peut susciter le besoin de simplification et de généralisation d'une forme au dépens des autres, cf . le pronom relatif, ou sa tendance à la disparition, cf. le passé simple. Mais «les modifications se font exclusivement dans le discursif » (SAUSSURE, 2002: 95). Toutefois, pour que ces variations relèvent de la dynamique synchronique, il faut qu'elles soient partagées par un certain nombre d'individus au sein de la communauté. Dès l'instant où elles sont acceptées par l'ensemble des locuteurs sur le long terme, elles se trouvent intégrées au système, qui se trouve, de ce fait transformé. Cette transformation ressortit alors à la diachronie.

\section{7. Conséquences et finalité}

Pour certains linguistes, notamment Frei et Martinet, toute variation a une finalité. Frei estime, en effet, que « loin de s'ajouter au langage comme un facteur externe, elle (l'idée de finalité) en constitue le principe et la raison d'être » (FREI, 1971 : 21). Il considère que ces variations peuvent répondre, selon les cas, à des besoins d'assimilation, de différenciation, de clarté, de brièveté, d'invariabilité, qui visent à la simplification et à l'économie, et d'expressivité.

Martinet, quant à lui, se refuse « à discuter... la question de savoir si l'on doit, en phonologie diachronique, parler de finalité ou de causalité » (MARTINET, 1970 : 17-18). Néanmoins, il considère que tout changement phonétique obéit d'une manière ou d'une autre au principe d'économie et qu'il satisfait généralement les besoins de communication, point de vue qui devrait pouvoir concerner les autres unités de la langue.

En réalité, il me paraît difficile d'interpréter l'ensemble des phénomènes de variation en termes de finalité, de même qu'il n'est pas évident d'identifier les causalités, ainsi que nous l'avons vu précédemment. Si la création d'unités nouvelles et leur formation par analogie répondant à l'arbitraire relatif de Saussure, cf. lave-linge, peut participer simultanément du besoin de 
communication et du principe d'économie, il n'en va pas nécessairement de même de toutes les variations. Ainsi l'emploi de $s u r^{7}$ au détriment de $a ̀$ dans une phrase extraite d'une interview à la radio, j'ai fait mes courses sur Grenoble, n'obéit a priori à aucun de ces facteurs, puisque la communication est assurée dans l'usage « standard » par à et que les deux prépositions sur et à coexistent dans le système. Cependant les variations liées à une concurrence des unités entre elles impliquent une réorganisation du système dans la mesure où les frontières entre ces unités changent, mais elles ne présupposent pas nécessairement une régulation du système, ni une économie lorsque leur existence respective n'est pas menacée, ni un progrès ou une détérioration de la langue. Comme l'affirme Bally, «nous sommes encore trop portés à confondre évolution et progrès, changement et amélioration » (BALLY, 1952 : 34). Il y a toujours «progrès et recul simultanés, mouvement de bascule, gain d'un côté, perte de l'autre » (BALLY, 1952 : 49).

\section{RAPPORTS ENTRE LES PHÉNOMÈNES D'OSCILLATION ET LES FAITS DE DYNAMIQUE EN SYNCHRONIE}

Les unités linguistiques en tant qu'entités systémiques sont, par nature, arbitraires, différentielles (SAUSSURE, 1916) et potentielles (MATHESIUS, 1911). Dans le discours, elles sont caractérisées par leurs oscillations (MATHESIUS, 1911) ou par leurs fluctuations (SAUSSURE, 2002 : 71-72), c'est-à-dire par leurs latitudes de réalisation au sein d'une valeur institutionnalisée. Or, ces variantes de réalisation peuvent conduire progressivement à la concurrence, voire au remplacement d'une unité par une autre (phonème, monème, fonction), d'une forme ou d'un effet de sens par une autre forme ou un autre effet de sens. À titre d'illustration, si deux phonèmes $\mathrm{A} / \tilde{\mathfrak{e}} /$ et $\mathrm{B} / \tilde{\varepsilon} /$ admettent plusieurs réalisations, il se peut que l'une des variantes de /A/ soit si proche de l'une des variantes de/B/ que celle-ci finisse par se substituer à celle de /A/. /A/ se trouvera alors en concurrence avec /B/ et pourra être éventuellement éliminé dans le temps.

Nous pouvons schématiser ci-après certains des phénomènes examinés.

\footnotetext{
${ }^{7}$ Voir également PARADIS, Gaële (2006), La dynamique des prépositions en et sur dans leur usage spatial en français contemporain, Thèse de Doctorat, sous la dir. de C. Feuillard, Paris, Université René Descartes-Paris 5. Cette étude a permis, entre autres, de mettre en évidence que les locuteurs ne faisaient pas de différence de sens entre les prépositions en et sur dans les usages émergents observés, et celles avec lesquelles elles étaient en concurrence, notamment dans et $\grave{a}$.
} 


\section{PHONÈMES}

\section{$I^{e r}$ stade $:$ oscillations}

Phonèmes
Variantes
de réalisation

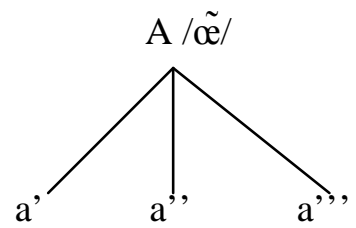

$\neq$

$\mathrm{B} / \tilde{\varepsilon} /$

b'

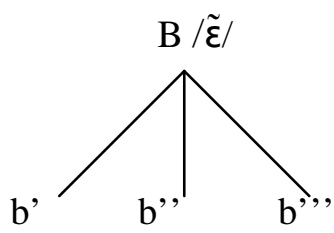

\section{$2^{\text {ème }}$ stade : dynamique synchronique}

Concurrence entre les unités au détriment de l'une d'elles


réalisation

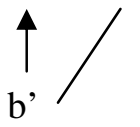

$\mathrm{B} / \tilde{\varepsilon} /$ empiète sur le domaine de A / $\tilde{\mathrm{e}} /$.

$\mathrm{B} / \tilde{\varepsilon} /$, usage extensif.

A /œ̃e/, usage récessif.

\section{$3^{\text {ème }}$ stade : dynamique diachronique}

Disparition de l'unité concurrencée A /ã//

Phonème

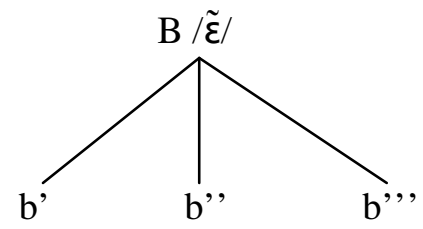

Variantes

de réalisation

b'

b',

b', 


\section{MONÈMES}

\section{$1^{e r}$ stade : oscillations ${ }^{8}$}

Monèmes

Contextes d'emploi

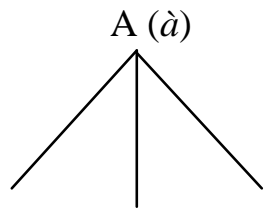

a' a', - à Grenoble - $\neq$

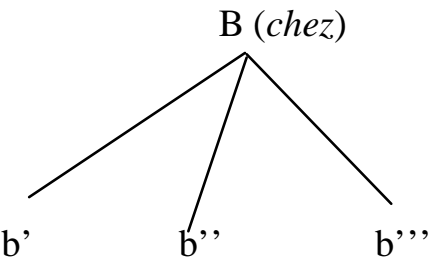

chez lui

Concurrence entre les unités au détriment de l'une d'elles

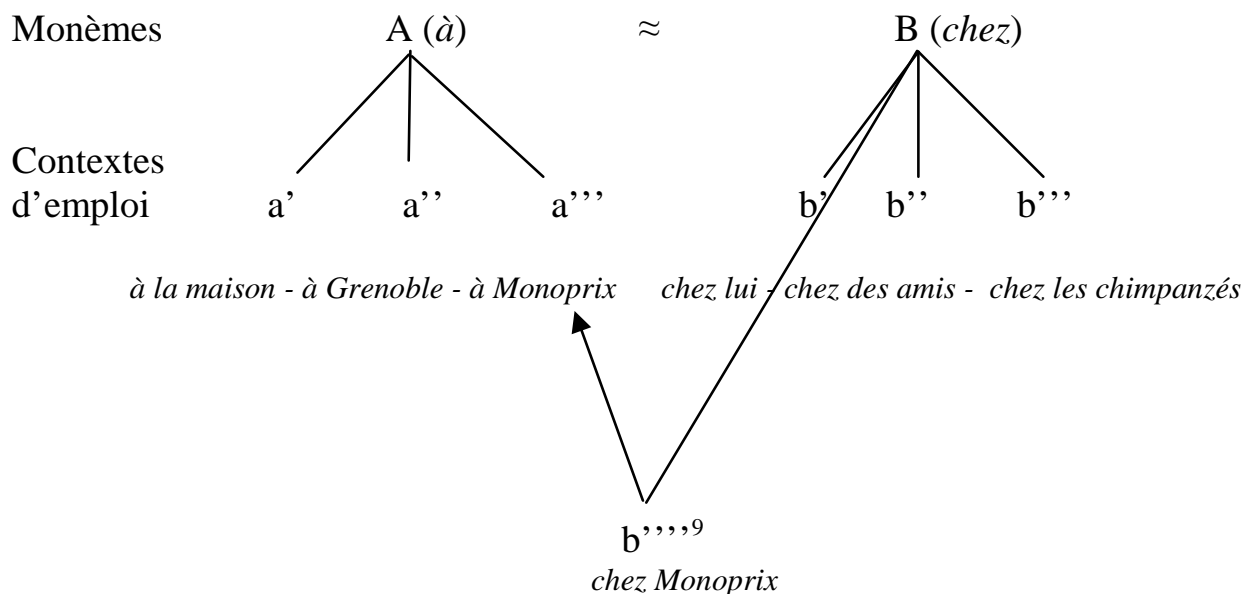

$\mathrm{A}(\grave{a})$ et $\mathrm{B}($ chez) coexistent dans le système.

$\mathrm{B}$ (chez) empiète sur le domaine de $\mathrm{A}(\grave{a})$ dans un contexte où $\mathrm{B}$ n'est pas admis par la norme standard. B : usage extensif.

Réduction d'emploi de A (à) dans un contexte au profit de B (chez). A : usage récessif.

\footnotetext{
${ }^{8}$ Comme il n'est pas possible, dans le cadre d'une étude aussi limitée, de faire une étude sémantique des prépositions examinées, nous simplifierons l'analyse en considérant que ces oscillations se manifestent dans les contextes d'emploi retenus.

${ }^{9}$ C'est l'apparition d'un nouveau contexte pour B (b",') qui justifie la légère différence de ce schéma avec celui qui a été proposé pour les phonèmes.
} 


\section{FONCTION SYNTAXIQUE}

\section{$I^{e r}$ stade : pas d'oscillations}

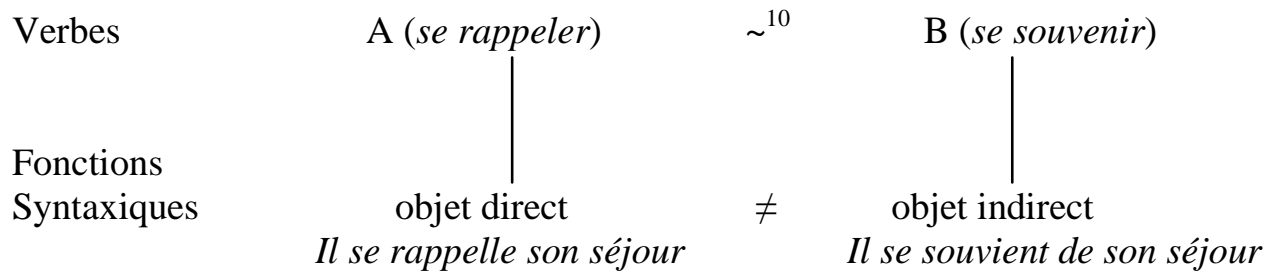

\section{$2^{\text {ème }}$ stade : dynamique synchronique}

Concurrence entre les fonctions au détriment de l'une d'elles

Verbes

Fonctions

Syntaxiques

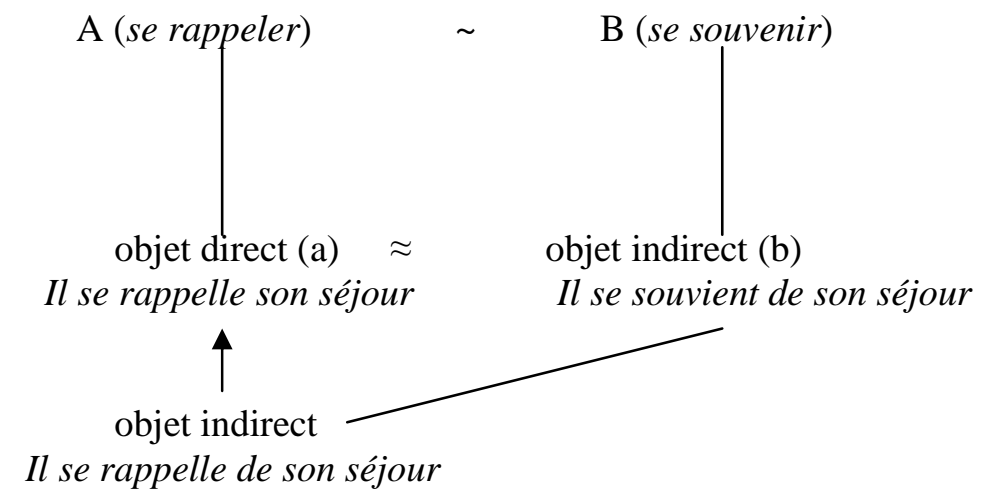

L'objet indirect (b) de B (se souvenir) concurrence l'objet direct (a) de A (se rappeler)

L'usage de (a) avec A est récessif.

L'usage de (b) est extensif, puisqu'il a tendance à se substituer à (a) avec A.

$$
3^{\text {ème }} \text { stade : dynamique diachronique } ?^{11}
$$
Monèmes
A (se rappeler)
$\sim \quad \mathrm{B}$ (se souvenir)
verbaux
Fonctions
Syntaxiques

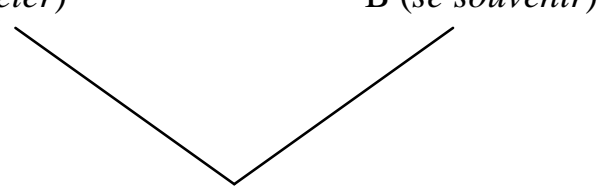
objet indirect (b)
Il se rappelle de son séjour
Il se souvient de son séjour

Disparition de l'usage de (a) avec A, remplacement par (b).

\footnotetext{
${ }^{10}$ Analogie de sens entre les deux unités.

${ }^{11}$ Il s'agit d'une simple hypothèse.
} 


\section{FORME}

Monème

\section{$1^{\text {er }}$ stade : oscillations}

de réalisation

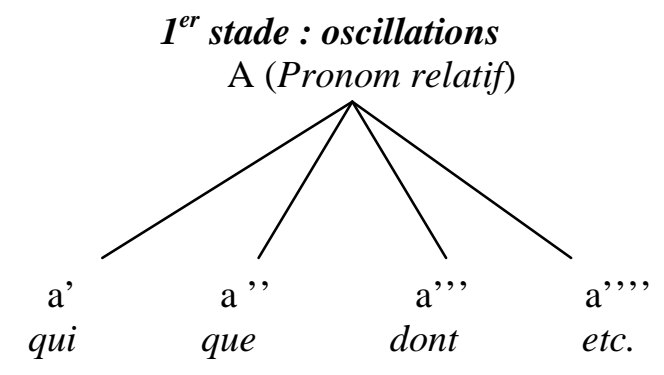

$2^{\text {ème }}$ stade : dynamique synchronique

Concurrence au profit de l'une des formes

Monème

Variantes

de réalisation

A (Pronom relatif)

Réalisation



$q u i$

a',

a',

a',,

dont

etc.

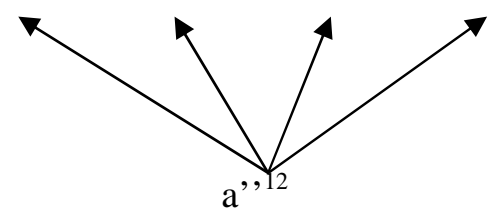

que

Les formes a' (qui), a',' (dont) sont récessives.

La forme a', (que) est en extension.

Monème

Réalisation

unique

\section{$3^{\text {ème }}$ stade : dynamique diachronique?}

A (Pronom relatif)

$\mathrm{a}^{\prime}$,

que

\footnotetext{
${ }^{12}$ Les différentes formes ont été représentées arbitrairement et sans souci de hiérarchie a', a', etc.
} 


\section{SENS}

\section{$I^{\text {er }}$ stade : oscillations}

Monème

Contextes

d'emploi

Effets

de sens

Hiérarchie

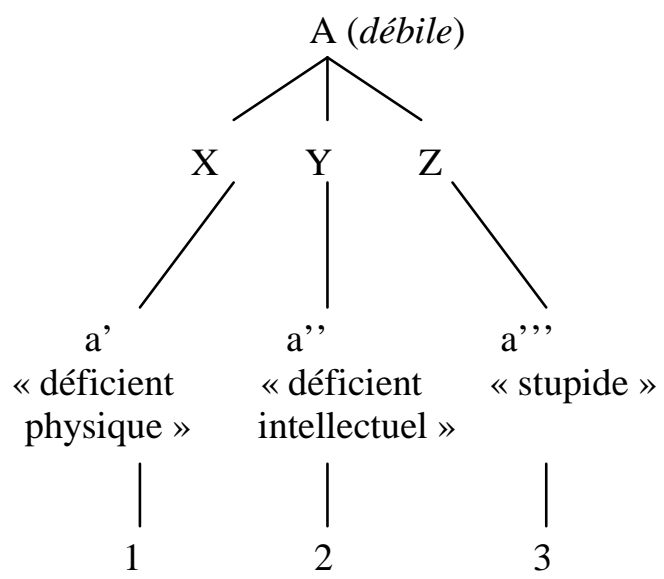

\section{$2^{\text {ème }}$ stade : dynamique synchronique}

Concurrence entre les effets de sens

Monème

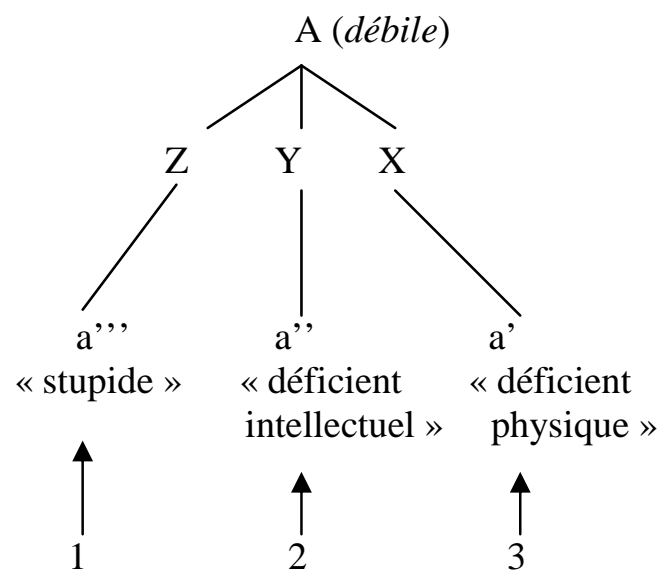

Hiérarchie

Contextes

d'emploi

Effets

de sens

Variation de la hiérarchie avec éventuellement disparition de certains effets de sens (a'?).

Ces schématisations ne rendent compte que des cas relevant de la concurrence entre les éléments et ne valent que pour les exemples analysés. Elles montrent, néanmoins, à l'exception des fonctions syntaxiques qui ne subissent pas en ellesmêmes d'oscillations que celles-ci sont en grande partie liées à de nombreux faits de dynamique synchronique et qu'elles peuvent en quelque sorte leur servir de « creuset». Ce lien entre oscillations et phénomènes de dynamique synchronique résulte du fait que la réalisation matérielle de la langue repose sur un continuum. 
Toutefois, l'on ne saurait assimiler oscillations et variations relevant de la dynamique synchronique. Ces dernières affectent l'identité linguistique des unités contrairement aux oscillations, et si les oscillations peuvent avoir une incidence sur la dynamique synchronique, tous les phénomènes de dynamique synchronique ne sauraient être reliés à des faits d'oscillations, tels, par exemple la variation de construction d'un verbe, se rappeler, ou la création de nouvelles unités. La dynamique synchronique recouvre donc un champ beaucoup plus large que les oscillations des unités.

\section{BIBLIOGRAPHIE}

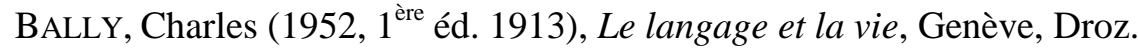

Feuillard, Colette \& PARADIS, Gaële (2002), La dynamique des relations spatiales, L'Imaginaire linguistique, (sous la dir. de Anne-Marie HoudebineGravaud), Paris, L'Harmattan.

FREI, Henri (1971, $1^{\text {ère }}$ éd. 1929), La grammaire des fautes, Genève, Slatkins Reprints.

HJEMSLEV, Louis (1971, $1^{\text {ère }}$ éd. 1943), Prolégomènes à une théorie du langage, Paris, éd. de Minuit.

LITTRE, Émile (1956-1958), Dictionnaire de la langue française, Paris, GallimardHachette.

MARTINET, André (1970, 1 ère éd. 1955), Économie des changements phonétiques, Berne, A. Francke.

MARTINET, André (1975), Évolution des langues et reconstruction, Paris, PUF.

MARTINET, André (1990), La synchronie dynamique, La Linguistique, Paris, PUF, Vol. 26/2.

Mathesius, Vilém (1911) On the Potentiality of the Phenomena of Language, dans A Prague School Reader in LINGUISTICS, compiled by Josef Vachek (1964), Indiana, Bloomington, University Press.

PARADIS, Gaële (2006), La dynamique des prépositions en et sur dans leur usage spatial en français contemporain, Thèse de Doctorat, sous la dir. de C. Feuillard, Paris, Université René Descartes.

SAUSSURE, Ferdinand (1972, $1^{\text {ère }}$ éd.1916), Cours de linguistique générale, Paris, Payot.

SAUSSURE, Ferdinand (2002), Écrits de linguistique générale, texte établi par Simon Bouquet \& Rudolf Engler, Paris, Gallimard.

Trésor de la langue française : http://atilf.fr/dendien/scripts/tlf...

\section{ABSTRACT}

The present paper aims to discuss the notions and phenomena of oscillation and potentiality (Mathesius) in comparison to synchronic dynamic of language (Martinet). The objective will be to see the field of each of them and their mutual relations. 